\title{
Effect of Sitagliptin on Serum Irisin Levels in Patients with Newly Diagnosed Type 2 Diabetes Mellitus
}

Qiu Wang · Lirong Ma · Yuanying Zhang · Lin Zhang •

Yu An · Jia Liu · Guang Wang (D)

Received: December 17, 2020 / Accepted: February 4, 2021 / Published online: February 24, 2021

(C) The Author(s) 2021

\section{ABSTRACT}

Introduction: Irisin is a unique myokine with striking effects on regulating insulin sensitivity and energy metabolism. This study aimed to investigate the changes in serum irisin in patients with newly diagnosed type 2 diabetes mellitus (T2DM) following sitagliptin treatment.

Methods: Thirty-two patients with T2DM were treated with $100 \mathrm{mg} /$ day sitagliptin for 16 weeks. Twenty age-, sex- and body mass index (BMI)-matched healthy subjects were enrolled as the control group. Irisin and metabolic parameters were measured at baseline and after treatment.

Qiu Wang and Lirong Ma contributed equally to this work.

Q. Wang · Y. Zhang · L. Zhang · Y. An · J. Liu (ه) .

G. Wang ( $\square)$

Department of Endocrinology, Beijing Chao-yang

Hospital, Capital Medical University, Beijing

100020, China

e-mail: liujia0116@126.com

G. Wang

e-mail: drwg6688@126.com

L. Ma · G. Wang

Department of Endocrinology, Beijing First Hospital of Integrated Chinese and Western Medicine,

Beijing 100026, China
Results: Patients with T2DM had lower irisin levels than the controls $(10.03 \pm 2.06$ vs. $13.06 \pm 3.10 \mathrm{ng} / \mathrm{ml}, P<0.01)$. Sitagliptin treatment significantly increased serum irisin levels in T2DM patients compared to baseline (11.18 \pm 1.91 vs. $10.03 \pm 2.06 \mathrm{ng} / \mathrm{ml}, \quad P<0.01)$. Increased irisin levels were associated with decreased fasting blood glucose (FBG) $(\beta=-0.24, P<0.05)$ and glycosylated hemoglobin (HbA1c) $(\beta=-0.15, P<0.05)$.

Conclusions: Sitagliptin treatment significantly increased serum irisin levels in patients with T2DM, and the increase of the irisin level was associated with decreases of FBG and HbA1c levels. These results suggest that irisin might be involved in the antidiabetic mechanisms of sitagliptin.

Trial Registration: ClinicalTrials.gov identifier NCT04495881. 
Keywords: Irisin; Sitagliptin; Type 2 diabetes mellitus

\section{Key Summary Points}

Type 2 diabetes mellitus (T2DM) has become a global epidemic, and decreased irisin levels are associated with metabolic disturbances of T2DM

Sitagliptin is used in treatment of T2DM and can upregulate the expression of irisin in a rat model of T2DM

Our study aimed to investigate the changes of serum irisin levels in patients with newly diagnosed T2DM following sitagliptin treatment

Sitagliptin treatment significantly increased serum irisin in patients with T2DM

Irisin might be involved in the antidiabetic mechanisms of sitagliptin

\section{DIGITAL FEATURES}

This article is published with digital features, including a summary slide, to facilitate understanding of the article. To view digital features for this article go to https://doi.org/10.6084/ m9.figshare.13712896.

\section{INTRODUCTION}

Irisin, a unique myokine, is regulated by peroxisome proliferator-activated receptor- $\gamma$ coactivator-1 $\alpha$ (PGC-1 $\alpha)$ and secreted primarily from skeletal muscle into the circulation [1]. It can promote energy expenditure and glucose uptake and reduce gluconeogenesis, lipogenesis and lipid accumulation through autocrine, paracrine or endocrine [2]. Abnormal serum irisin levels have been shown to associate with multiple metabolic diseases including obesity [3], type 2 diabetes mellitus (T2DM) [4], gestational diabetes mellitus (GDM) [5], nonalcoholic fatty liver disease (NAFLD) [6], metabolic syndrome [7] and polycystic ovary syndrome (PCOS) [8].

T2DM is a chronic metabolic disorder characterized by hyperglycemia, insulin resistance and relative insulin deficiency [9]. Sitagliptin is a representative of dipeptidyl peptidase-4 (DPP4) inhibitors, which are effectively used in the treatment of T2DM [10]. Recently, some animal studies have shown that sitagliptin upregulated the expression of PGC- $1 \alpha$ and irisin to attenuate insulin resistance, improve glycolipid metabolism and inhibit inflammation in a T2DM rat model [11]. However, up to now, there are few reports regarding the effects of sitagliptin on serum irisin levels in patients with T2DM.

Therefore, our present study aimed to investigate the changes of serum irisin in patients with newly diagnosed T2DM after sitagliptin treatment.

\section{METHODS}

\section{Study Design and Subjects}

Between May 2020 and July 2020, we consecutively recruited 32 patients with newly diagnosed type 2 diabetes mellitus (T2DM group) and 20 age-, sex- and BMI-matched healthy subjects (control group) who received routine physical examination from the outpatients at the Department of Endocrinology in Beijing Chao-yang Hospital affiliated with Capital Medical University. The patients aged 21-65 years with newly diagnosed T2DM within the previous 3 months met the following inclusion criteria: (1) the diagnosis of T2DM according to the 2014 American Diabetes Association (ADA) diagnostic criteria and (2) HbA1c $\geq 7.0 \%$ and $\leq 9.5 \%$ [12]. Meanwhile, they did not receive any oral hypoglycemic drugs before the study enrollment. The healthy controls had a normal glucose tolerance via an oral glucose tolerance test (OGTT). All patients with type 1 diabetes mellitus, pancreatitis, pregnancy or possible pregnancy, liver or renal function impairment, coronary heart disease, infectious disease or cancer were excluded. The 
T2DM patients received $100 \mathrm{mg}$ sitagliptin orally once daily for 16 weeks. Furthermore, lifestyle modifications (diet and physical activity) were delivered to all patients, and lifestyle information was collected through telephone contact.

The study was registered at Clinicaltrials.gov (NCT04495881) and was conducted in compliance with the Declaration of Helsinki. The protocol was approved by the Ethics Committee of Beijing Chao-yang Hospital affiliated with Capital Medical University (2020). Written informed consent was obtained from all participants in this study.

\section{Clinical and Biochemical Measurements}

All enrolled patients were followed up for 16 weeks. Before and after the 16-week intervention, fasting blood samples were obtained in the morning after the overnight fasting, and serum samples were stored at $-80^{\circ} \mathrm{C}$ until analysis. During the follow-up period, hypoglycemia did not occur in the T2DM group, but six patients dropped out of the study because of poor glucose control.

Anthropometric and biochemical measurements were collected at baseline and after 16 weeks of sitagliptin treatment. Height and body weight were measured to the nearest $0.1 \mathrm{~cm}$ and $0.1 \mathrm{~kg}$, respectively. Total cholesterol (TC), low-density lipoprotein cholesterol (LDL-C), high-density lipoprotein cholesterol (HDL-C) and triglycerides (TG), FBG, fasting insulin (FINS) and HbA1c were analyzed at the central chemistry laboratory of Beijing Chaoyang Hospital affiliated with Capital Medical University. Moreover, serum irisin levels were measured using enzyme-linked immunosorbent assay (ELISA) kits (EK-067-29, Phoenix Pharmaceuticals, Inc., Burlingame, CA, USA) for quantitative detection. The sensitivity of the assay was $2.42 \mathrm{ng} / \mathrm{ml}$, and the linear range of the standard was $2.42-23.30 \mathrm{ng} / \mathrm{ml}$. The BMI was calculated as body weight divided by height squared $\left(\mathrm{kg} / \mathrm{m}^{2}\right)$. Homeostasis model assessment of insulin resistance (HOMA-IR) was calculated as FBG $(\mathrm{mmol} / \mathrm{l}) \times$ FINS $(\mu \mathrm{IU} / \mathrm{ml}) / 22.5$, and homeostatic model assessment of $\beta$-cell function $($ HOMA- $\beta$ ) was calculated as $20 \times$ FINS $(\mu \mathrm{IU} / \mathrm{ml}) /[\mathrm{FBG}(\mathrm{mmol} / \mathrm{l})-3.5][13,14]$.

\section{Statistical Analysis}

Statistical analyses were performed using the SPSS statistical software, version 23.0 (SPSS Inc., Chicago, IL, USA). One-sample Kolmogorov-Smirnov test was used to confirm the normality of distribution. The data were presented as means \pm standard deviation (SD) or median (interquartile range). The differences between healthy controls and T2DM patients were tested by the Student's $t$-test or nonparametric Mann-Whitney $U$ test. The paired Student's $t$-test or nonparametric Wilcoxon test was used to compare statistical differences between pre- and post-treatment. The differences in proportions were analyzed using the chi-square test. The Pearson or Spearman correlation coefficient was used for the correlations between serum irisin levels and metabolic parameters at baseline as appropriate. Linear mixed effects models were performed using STATA version 13.0 (STATA, College Station, TX) to account for the longitudinal relationship between serum irisin levels and glucose-lipid metabolic parameters over a 16-week period. All statistical tests were two-tailed, and $P$ values $<0.05$ were considered statistically significant.

\section{RESULTS}

\section{Baseline Clinical Characteristics of the Study Participants}

The baseline clinical characteristics of the T2DM and control group are listed in Table 1. The participants in both groups were similar in age, sex, BMI, TC, LDL-C, HDL-C, TG and FINS (all $P>0.05$ ). However, the T2DM patients had significantly higher FBG, HbA1c and HOMA-IR and lower HOMA- $\beta$ levels than the control subjects (all $P<0.01$ ). The serum irisin levels were significantly lower in the T2DM group than in the control group $(10.03 \pm 2.06$ vs. $13.06 \pm 3.10 \mathrm{ng} / \mathrm{ml}, P<0.01)$. 
Table 1 Baseline characteristics of the study participants

\begin{tabular}{|c|c|c|c|}
\hline & \multicolumn{2}{|l|}{ Groups } & \multirow[t]{2}{*}{$P$ value } \\
\hline & $\begin{array}{l}\text { Matched } \\
\text { control } \\
(n=20)\end{array}$ & $\begin{array}{l}\text { Type } 2 \text { diabetes } \\
\text { mellitus } \\
(n=32)\end{array}$ & \\
\hline $\begin{array}{l}\text { Age, } \\
\text { years }\end{array}$ & $46.00 \pm 13.54$ & $49.63 \pm 13.83$ & 0.358 \\
\hline $\begin{array}{l}\text { Sex, } \\
\text { M/F }\end{array}$ & $12 / 8$ & $20 / 12$ & 0.857 \\
\hline $\begin{array}{l}\text { BMI, } \\
\mathrm{kg} / \mathrm{m}^{2}\end{array}$ & $25.24 \pm 3.59$ & $26.16 \pm 3.44$ & 0.359 \\
\hline $\begin{array}{l}\text { TC, } \\
\text { mmol/ } \\
1\end{array}$ & $4.77 \pm 0.96$ & $4.79 \pm 0.91$ & 0.944 \\
\hline $\begin{array}{l}\text { LDL-C, } \\
\text { mmol/ } \\
1\end{array}$ & $2.83 \pm 1.01$ & $3.07 \pm 0.92$ & 0.385 \\
\hline $\begin{array}{l}\text { HDL-C, } \\
\text { mmol/ } \\
1\end{array}$ & $1.30 \pm 0.37$ & $1.14 \pm 0.26$ & 0.098 \\
\hline $\begin{array}{l}\text { TG, } \\
\qquad \mathrm{mmol} / \\
1\end{array}$ & $\begin{array}{c}1.46(0.94 \\
2.07)\end{array}$ & $1.72(1.20,2.25)$ & 0.328 \\
\hline $\begin{array}{l}\text { FBG, } \\
\qquad \mathrm{mmol} / \\
1\end{array}$ & $4.97 \pm 0.59$ & $8.51 \pm 1.71$ & $<0.001^{*}$ \\
\hline $\begin{array}{l}\text { FINS, } \\
\begin{array}{l}\mu \mathrm{IU} / \\
\mathrm{ml}\end{array}\end{array}$ & $\begin{array}{c}7.25(4.90 \\
13.33)\end{array}$ & $9.65(6.15,14.85)$ & 0.310 \\
\hline $\begin{array}{l}\mathrm{HbAlc} \\
\%\end{array}$ & $5.56 \pm 0.47$ & $8.05 \pm 0.81$ & $<0.001^{*}$ \\
\hline $\begin{array}{l}\text { HOMA- } \\
\text { IR }\end{array}$ & $\begin{array}{l}1.65(1.00 \\
3.04)\end{array}$ & $3.51(2.03,5.62)$ & $0.003^{*}$ \\
\hline $\begin{array}{c}\text { HOMA- } \\
\beta\end{array}$ & $\begin{array}{l}114.14(70.06 \\
128.14)\end{array}$ & $\begin{array}{c}40.83(23.06 \\
58.33)\end{array}$ & $<0.001^{*}$ \\
\hline
\end{tabular}

Table 1 continued

\begin{tabular}{|c|c|c|c|}
\hline & \multicolumn{2}{|l|}{ Groups } & \multirow{2}{*}{$P$ value } \\
\hline & $\begin{array}{l}\text { Matched } \\
\text { control } \\
(n=20)\end{array}$ & $\begin{array}{l}\text { Type } 2 \text { diabetes } \\
\text { mellitus } \\
(n=32)\end{array}$ & \\
\hline $\begin{array}{l}\text { Irisin, } \\
\mathrm{ng} / \mathrm{ml}\end{array}$ & $13.06 \pm 3.10$ & $10.03 \pm 2.06$ & $0.001^{*}$ \\
\hline
\end{tabular}

Data shown as mean \pm standard deviation were compared between two groups using Student's $t$-test for independent samples

Data shown as median (interquartile range) were compared between two groups using Mann-Whitney $U$ test

Data shown as $n$ (\%) were compared between two groups using the chi-square test

$B M I$ body mass index, $T C$ total cholesterol, $L D L-C$ lowdensity lipoprotein cholesterol, $H D L-C$ high-density lipoprotein cholesterol, $T G$ triglyceride, $F B G$ fasting blood glucose, FINS fasting insulin, HbAIc glycosylated hemoglobin, HOMA-IR homeostasis model assessment of insulin resistance, HOMA- $\beta$ homeostasis model assessment of $\beta$-cell function

${ }^{*} P<0.05$

\section{Correlations Between Serum Irisin Levels and the Baseline Parameters}

We investigated the correlations between serum irisin levels and the baseline metabolic parameters in both groups. The serum irisin levels were found to be negatively correlated with TG, FBG and HbA1c (TG: $r=-0.291, P<0.05$; FBG: $r=-0.431, \quad P<0.01 ; \quad$ HbA1c: $r=-0.338$, $P<0.05)$. Besides, serum irisin levels were significantly positively correlated with HDL-C and HOMA- $\beta$ (HDL-C: $r=0.280, P<0.05$; HOMA- $\beta$ : $r=0.323, P<0.05$ ) (Table 2).

\section{Effect of Sitagliptin Treatment on Metabolic Parameters and Serum Irisin Levels in T2DM Patients}

The observed changes in metabolic parameters after sitagliptin treatment in the T2DM group are summarized in Table 3. The levels of BMI, FBG, HbA1c and HOMA-IR were significantly decreased from baseline after sitagliptin 
Table 2 Correlation analyses of serum irisin levels with biochemical parameters in baseline

\begin{tabular}{lrl}
\hline & \multicolumn{1}{l}{ Irisin } & \multicolumn{1}{r}{$\boldsymbol{P}$ value } \\
\cline { 2 - 3 } & $\boldsymbol{r}$ & 0.797 \\
\hline Age & -0.037 & 0.643 \\
BMI & -0.066 & 0.591 \\
TC & 0.076 & 0.588 \\
LDL-C & 0.077 & $0.044^{*}$ \\
HDL-C & 0.280 & $0.036^{*}$ \\
TG & -0.291 & $0.001^{*}$ \\
FBG & -0.431 & 0.493 \\
FINS & -0.097 & $0.002^{*}$ \\
HbA1c & -0.442 & 0.123 \\
HOMA-IR & -0.216 & $0.020^{*}$ \\
HOMA- $\beta$ & 0.323 &
\end{tabular}

$B M I$ body mass index, $T C$ total cholesterol, $L D L-C$ lowdensity lipoprotein cholesterol, $H D L-C$ high-density lipoprotein cholesterol, $T G$ triglyceride, $F B G$ fasting blood glucose, FINS fasting insulin, HbAlc glycosylated hemoglobin, HOMA-IR homeostasis model assessment of insulin resistance, HOMA- $\beta$ homeostasis model assessment of $\beta$-cell function

${ }^{*} P<0.05$

treatment in T2DM patients (all $P<0.01$ ). Increases in HOMA- $\beta$ were observed after sitagliptin treatment. However, there were no obvious changes in TC, LDL-C, HDL-C, TG and FINS (all $P>0.05$ ). Simultaneously, sitagliptin treatment also significantly increased serum irisin levels compared to baseline $(11.18 \pm 1.91$ vs. $10.03 \pm 2.06 \mathrm{ng} / \mathrm{ml}, P<0.01)$.

\section{Associations Between Serum Irisin and Metabolic Parameters after Sitagliptin Treatment}

Longitudinal analysis was performed by linear mixed effects models, and the data are displayed in Figs. 1 and 2. In multivariable models adjusted for sex, age, BMI and lipid profiles, higher serum irisin levels were associated with lower
FBG $(\beta=-0.24, P<0.05$, Fig. 1a) and lower HbA1c $(\beta=-0.15, P<0.05$, Fig. 1b). Using other regression models adjusted for sex, age, BMI, FBG, HbA1c and FINS, lipid metabolic parameters (TC, HDL-C, LDL-C and TG) were not significantly associated with serum irisin levels (all $P>0.05$, Fig. $2 \mathrm{a}-\mathrm{d}$ ).

\section{DISCUSSION}

In the present study, patients with newly diagnosed T2DM had significantly lower serum irisin levels than the controls. At baseline, serum irisin levels were negatively correlated with TG, FBG and HbA1c while positively correlated with HDL-C and HOMA- $\beta$. Sitagliptin treatment significantly increased serum irisin levels, accordance with the improvement of metabolic parameters. The observed increases of serum irisin after sitagliptin treatment were associated with the decreases of FBG and HbA1c.

Irisin is an exercise-mediated myokine. Irisin secretion is induced by exercise, which stimulates the expression of PGC- $1 \alpha$ and fibronectin type III domain-containing protein 5 (FNDC5) in skeletal muscles; thereafter, FNDC5 is cleaved, and the remaining 112-amino-acid peptide is known as irisin [1]. Some studies reported that circulating irisin levels decreased in newly diagnosed T2DM and long-term T2DM, which indicates that irisin may be a good biomarker of T2DM [4, 15-17]. We also found that the serum irisin levels were lower in patients with T2DM than in the control group. Moreover, consistent with other studies, serum irisin levels were negatively correlated with TG, FBG and HbA1c while positively correlated with HDL-C and HOMA- $\beta$ [4, 15, 18]. Mechanistically, it has been reported that irisin exerts metabolic benefits via distinct mechanisms. First, irisin activates the expression of uncoupling protein-1 (UCP-1) to accelerate the browning of white adipose tissue (WAT), which can increase energy consumption, improve glucose homeostasis and reduce insulin resistance $[19,20]$. Second, irisin can facilitate glucolipid metabolism through the upregulation of the expressions of glucose transporter type 4 (GLUT4), hexokinase 2 (HK2) and peroxisome 
Table 3 Pre- and post-treatment clinical characteristics of T2DM patients treated with sitagliptin

\begin{tabular}{|c|c|c|c|c|}
\hline & \multicolumn{2}{|l|}{ Group } & \multirow[t]{2}{*}{ Changes after sitagliptin } & \multirow[t]{2}{*}{$P$ value } \\
\hline & Pre-treatment $(n=32)$ & Post-treatment $(n=32)$ & & \\
\hline $\mathrm{BMI}, \mathrm{kg} / \mathrm{m}^{2}$ & $26.16 \pm 3.44$ & $25.69 \pm 3.58$ & $-0.47(-0.79,-0.16)$ & $0.005^{*}$ \\
\hline $\mathrm{TC}, \mathrm{mmol} / \mathrm{l}$ & $4.79 \pm 0.91$ & $4.73 \pm 0.83$ & $-0.06(-0.26,0.15)$ & 0.572 \\
\hline LDL-C, mmol/l & $3.07 \pm 0.92$ & $3.04 \pm 0.93$ & $-0.03(-0.27,0.21)$ & 0.813 \\
\hline HDL-C, $\mathrm{mmol} / \mathrm{l}$ & $1.14 \pm 0.26$ & $1.19 \pm 0.33$ & $0.05(-0.01,0.11)$ & 0.075 \\
\hline $\mathrm{TG}, \mathrm{mmol} / \mathrm{l}$ & $1.72(1.20,2.25)$ & $1.37(0.98,2.33)$ & $-0.09(-0.46,0.28)$ & 0.114 \\
\hline $\mathrm{FBG}, \mathrm{mmol} / \mathrm{l}$ & $8.51 \pm 1.71$ & $6.66 \pm 1.17$ & $-1.85(-2.38,-1.31)$ & $<0.001^{*}$ \\
\hline FINS, $\mu \mathrm{IU} / \mathrm{ml}$ & $9.65(6.15,14.85)$ & $9.65(5.63,12.85)$ & $-0.17(-1.75,1.40)$ & 0.888 \\
\hline HbAlc, \% & $8.05 \pm 0.81$ & $6.61 \pm 0.62$ & $-1.44(-1.75,-1.13)$ & $<0.001^{*}$ \\
\hline HOMA-IR & $3.51(2.03,5.62)$ & $2.70(1.67,3.99)$ & $-0.87(-1.43,-0.31)$ & $0.006^{*}$ \\
\hline HOMA- $\beta$ & $40.83(23.06,58.33)$ & $66.92(35.57,87.86)$ & $24.30(13.47,35.14)$ & $<0.001^{*}$ \\
\hline Irisin, $\mathrm{ng} / \mathrm{ml}$ & $10.03 \pm 2.06$ & $11.18 \pm 1.91$ & $1.15(0.70,1.60)$ & $<0.001^{*}$ \\
\hline
\end{tabular}

Data shown as mean \pm standard deviation were compared between pre- and post-treatment using paired Student's $t$-test Data shown as median (interquartile range) were compared between pre- and post-treatment using paired Wilcoxon test $B M I$ body mass index, $T C$ total cholesterol, $L D L-C$ low-density lipoprotein cholesterol, $H D L-C$ high-density lipoprotein cholesterol, $T G$ triglyceride, FBG fasting blood glucose, FINS fasting insulin, HbAlc glycosylated hemoglobin, HOMA-IR homeostasis model assessment of insulin resistance, HOMA- $\beta$ homeostasis model assessment of $\beta$-cell function ${ }^{*} P<0.05$

proliferator activated receptor $\alpha(\operatorname{PPAR} \alpha)$ and the stimulation of AMP-activated protein kinase (AMPK) phosphorylation in primary human skeletal muscle cell (HSMCs) [21]. Thus, these results suggest decreased irisin levels might be involved in the development of T2DM.

Consistent with other studies, sitagliptin treatment significantly decreased BMI, FBG, HbA1c and HOMA-IR and increased HOMA- $\beta$ in T2DM patients. However, there was a greater reduction of BMI and $\mathrm{HbA1c}$ in this study than in previous studies [22-24]. We believed that two reasons might explain these differences. On the one hand, patients with T2DM received sitagliptin as the initial therapy. In parallel, lifestyle modifications were used as an adjunctive therapy. On the other hand, sitagliptin significantly lowers postprandial glucose levels by stimulating the glucose-induced insulin secretion and inhibiting postprandial glucagon release, thus lowering higher postprandial plasma glucose levels in Chinese patients with T2DM $[25,26]$. Besides, our study showed that sitagliptin treatment significantly decreased HOMA-IR and increased HOMA- $\beta$. These results are supported by rodent studies showing that DPP-4 inhibitors promote $\beta$-cell proliferation and inhibit apoptosis $[27,28]$.

In this study, sitagliptin treatment significantly increased serum irisin levels, which were parallel with improving FBG and HbA1c. The results were also supported by the results of animal studies. In high-fat diet-fed mice,

Fig. 1 Forest plots demonstrating associations between irisin and FBG (a), HbAlc (b), HOMA-IR (c), HOMA- $\beta$ (d) and FINS (e) in mixed-effect linear models adjusted for potential confounders. $F B G$ fasting blood glucose, $H b A 1 c$ glycosylated hemoglobin, HOMA-IR homeostasis model assessment of insulin resistance, HOMA- $\beta$ homeostasis model assessment of $\beta$-cell function, FINS fasting insulin, $B M I$ body mass index, $T C$ total cholesterol, $H D L$ $C$ high-density lipoprotein cholesterol, $L D L-C$ low-density lipoprotein cholesterol, $T G$ triglyceride 


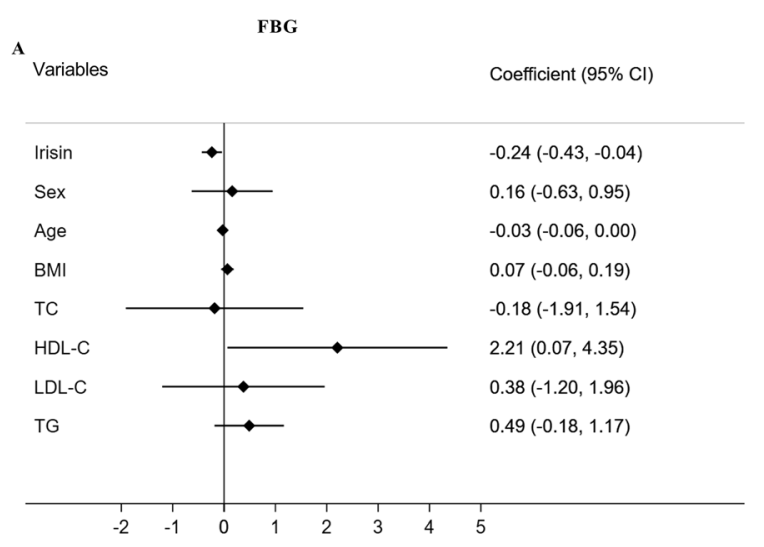

B

HbAlc

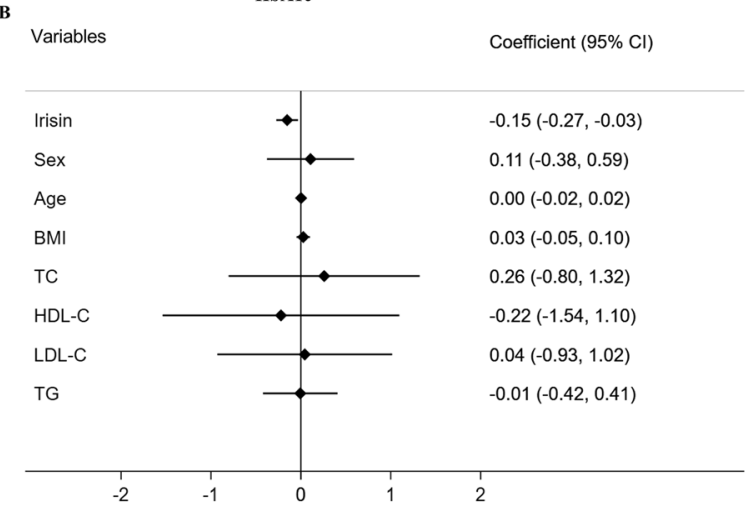

C

HOMA-IR
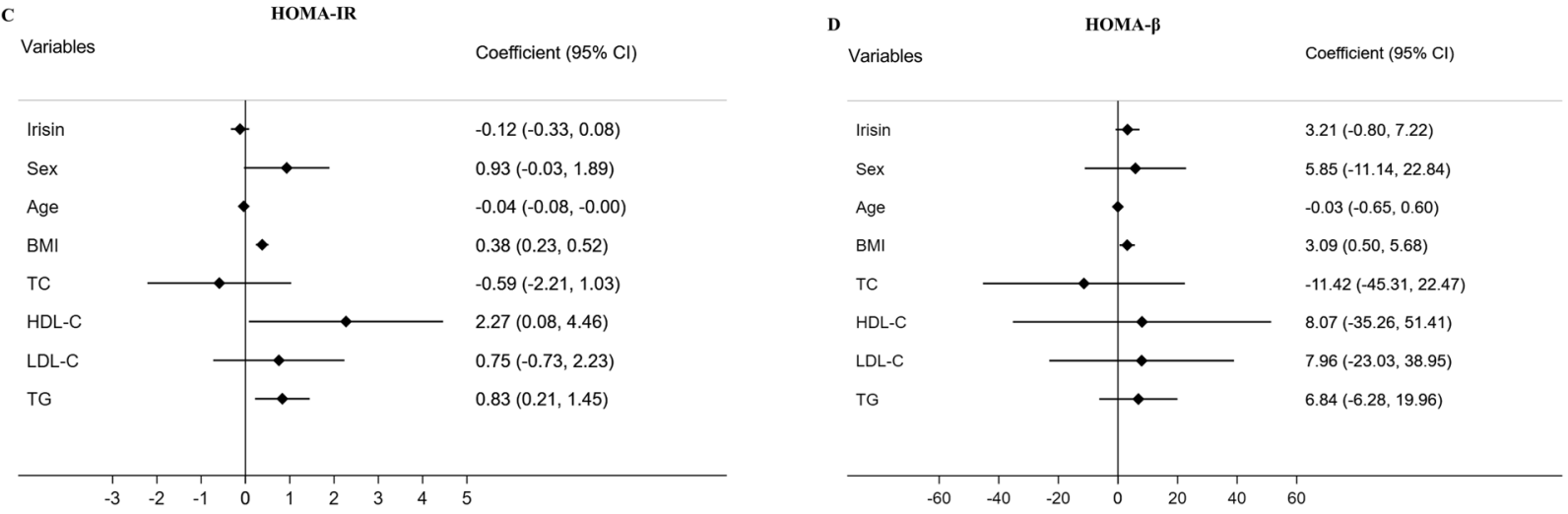

E

FINS

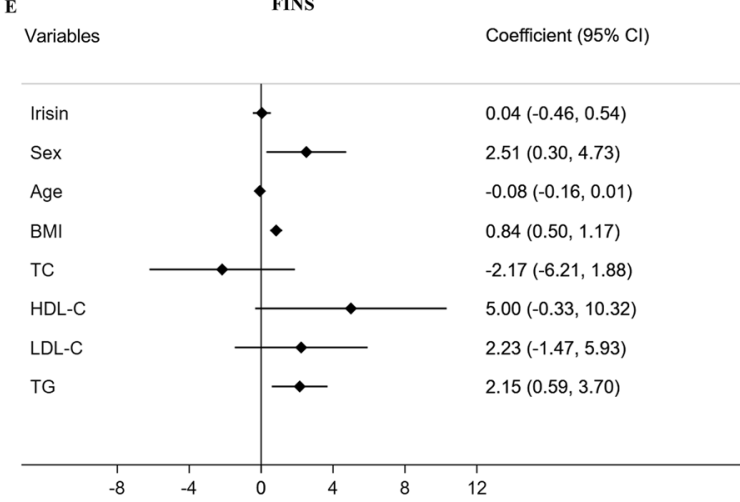


increased levels of circulating irisin, induced by adenoviral overexpression of FNDC5, substantially improved glucose tolerance and attenuated insulin resistance [1]. Then, irisin treatment increased glucose and fatty acid uptake, decreased the body weight and improved glucose metabolism via AMPK pathway $[20,29,30]$. In addition, irisin can increase the expression of betatrophin, another novel hormone that stimulates $\beta$-cell proliferation to improve glucose tolerance [20]. Therefore, the metabolic benefits of irisin suggested that irisin might participate in beneficial effects of sitagliptin in patients with T2DM. The exact mechanisms by which sitagliptin treatment increases serum irisin levels have been reported
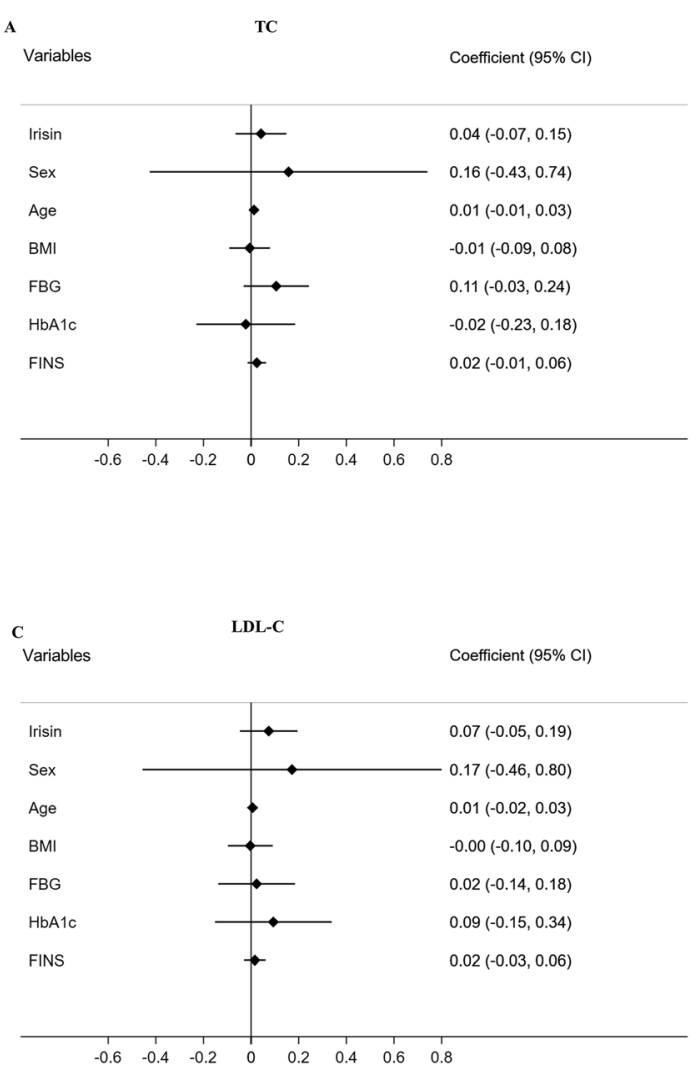

Fig. 2 Forest plots demonstrating associations between irisin and TC (a), HDL-C (b), LDL-C (c) and TG (d) in mixed-effect linear models adjusted for potential confounders. $T C$ total cholesterol, $H D L-C$ high-density lipoprotein cholesterol, $L D L-C$ low-density lipoprotein as follows: First, in a rat model of T2DM, sitagliptin significantly lowered the levels of fasting plasma glucose, FINS, TNF- $\alpha$, TC, TG and HOMA-IR. Further mechanism studies found that sitagliptin dose-dependently upregulated the protein expressions of PGC- $1 \alpha$, irisin and p-AMPK and the mRNA expressions of PGC- $1 \alpha$ and FNDC5 in skeletal muscle. In summary, sitagliptin improved insulin resistance and metabolic disruption produced by T2DM through the PGC- $1 \alpha$-irisin-p-AMPK pathway [11].

Our present study has several limitations. First, the study design was not a randomized controlled trial, so it might introduce bias into the results. Further well-designed randomized
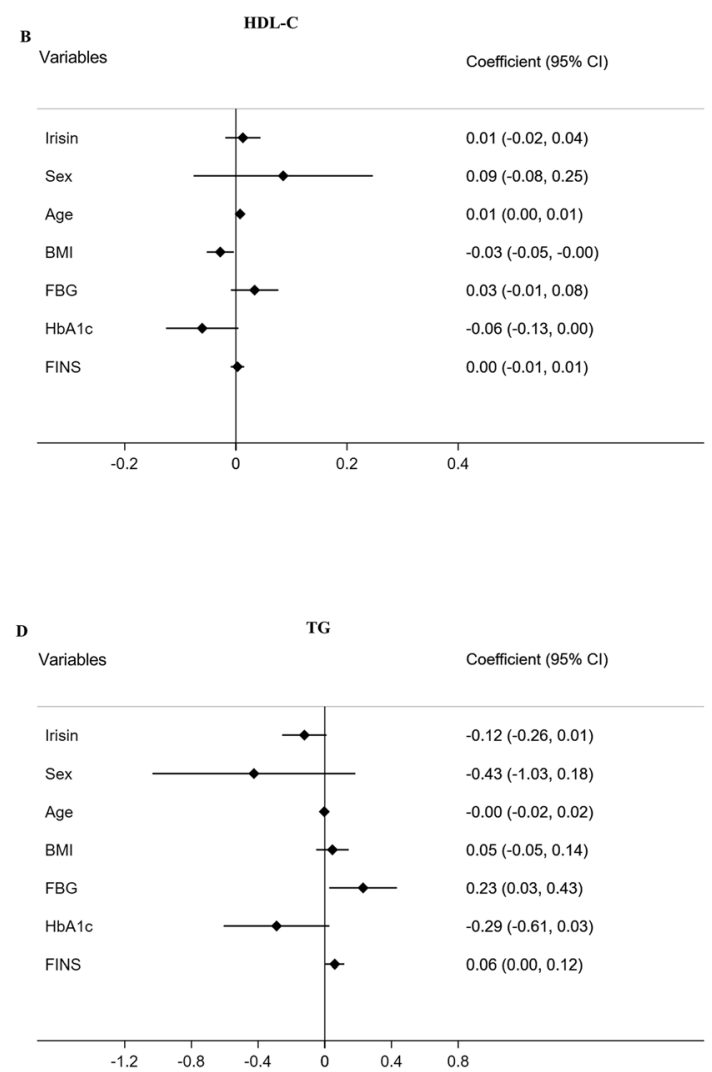

cholesterol, $T G$ triglyceride, $B M I$ body mass index, $F B G$ fasting blood glucose, $H b A l c$ glycosylated hemoglobin, FINS fasting insulin 
controlled trials are needed to confirm our results. Second, our results might be limited by the relatively small sample size; hence, a larger sample size is needed for further study. Moreover, lifestyle intervention is a cornerstone in first-line therapy of T2DM. Based on the treatment guidelines, we cannot exclude the influence of lifestyle modifications on the therapeutic efficacy of sitagliptin treatment. Many studies have shown that lifestyle modifications, especially exercise, can increase the circulating levels of irisin $[21,31,32]$. We did not systematically evaluate lifestyle modifications in T2DM patients, so we cannot exclude the effect of lifestyle modifications on irisin. Subsequent research should assess the effects of physical activity and diet modification. Nonetheless, this study has clinical significance as a pilot study to generate a hypothesis on the effect of irisin on the metabolic improvements by sitagliptin. The findings of our study are relevant to clinical practice in real world settings and have sufficient reliability based on a sophisticated statistical method.

\section{CONCLUSION}

In conclusion, our study demonstrated that the serum irisin levels were significantly decreased in patients with newly diagnosed T2DM. Sitagliptin treatment significantly increased serum irisin levels in patients with T2DM, and the increase in irisin was associated with the decreases in FBG and HbA1c levels. These results suggest that irisin might be involved in the antidiabetic mechanisms of sitagliptin.

\section{ACKNOWLEDGEMENTS}

We thank the participants of the study.

Funding. This study and the fee for the journal's Rapid Service were supported by the Chinese National Natural Science Foundation [no. 81770792] and Key Projects of Science and Technology Planning of Beijing Municipal Education Commission (KZ201810025038) to
Guang Wang and the Beijing Talents foundation (2018-12) to Jia Liu.

Authorship. All named authors meet the International Committee of Medical Journal Editors (ICMJE) criteria for authorship for this article, take responsibility for the integrity of the work as a whole, and have given their approval for this version to be published.

Author Contributions. GW and JL conceived and designed the study; QW and LM conducted the experiments, performed the analyses and wrote the manuscript. YZ, LZ and YA helped collect and analyze the data. All authors read and approved the final manuscript.

Disclosures. Qiu Wang, Lirong Ma, Yuanying Zhang, Lin Zhang, Yu An, Jia Liu and Guang Wang have nothing to disclose.

Compliance with Ethics Guidelines. The study was registered at Clinicaltrials.gov (NCT04495881) and was conducted in compliance with the Declaration of Helsinki. The protocol was approved by the Ethics Committee of Beijing Chao-yang Hospital affiliated with Capital Medical University (2020-\#-182). Written informed consent was obtained from all participants in this study. Full details relating to consent for publication.

Data Availability. The datasets generated or analyzed during the current study are available from the corresponding author on reasonable request.

Open Access. This article is licensed under a Creative Commons Attribution-NonCommercial 4.0 International License, which permits any non-commercial use, sharing, adaptation, distribution and reproduction in any medium or format, as long as you give appropriate credit to the original author(s) and the source, provide a link to the Creative Commons licence, and indicate if changes were made. The images or other third party material in this article are included in the article's Creative Commons licence, unless indicated otherwise in a credit 
line to the material. If material is not included in the article's Creative Commons licence and your intended use is not permitted by statutory regulation or exceeds the permitted use, you will need to obtain permission directly from the copyright holder. To view a copy of this licence, visit http://creativecommons.org/licenses/bync/4.0/.

\section{REFERENCES}

1. Boström P, Wu J, Jedrychowski MP, et al. A PGC1- $\alpha-$ dependent myokine that drives brown-fat-like development of white fat and thermogenesis. Nature. 2012;481:463-8.

2. Perakakis N, Triantafyllou GA, Fernández-Real JM, et al. Physiology and role of irisin in glucose homeostasis. Nat Rev Endocrinol. 2017;13(6): 324-37.

3. Moreno-Navarrete JM, Ortega F, Serrano M, et al. Irisin is expressed and produced by human muscle and adipose tissue in association with obesity and insulin resistance. J Clin Endocrinol Metab. 2013;98(4):E769-78.

4. Choi YK, Kim MK, Bae KH, et al. Serum irisin levels in new-onset type 2 diabetes. Diabetes Res Clin Pract. 2013;100(1):96-101.

5. Yuksel MA, Oncul M, Tuten A, et al. Maternal serum and fetal cord blood irisin levels in gestational diabetes mellitus. Diabetes Res Clin Pract. 2014;104(1):171-5.

6. Polyzos SA, Kountouras J, Anastasilakis AD, Geladari EV, Mantzoros CS. Irisin in patients with nonalcoholic fatty liver disease. Metabolism. 2014;63(2):207-17.

7. Park KH, Zaichenko L, Brinkoetter M, et al. Circulating irisin in relation to insulin resistance and the metabolic syndrome. J Clin Endocrinol Metab. 2013;98(12):4899-907.

8. Chang CL, Huang SY, Soong YK, Cheng PJ, Wang CJ, Liang IT. Circulating irisin and glucose-dependent insulinotropic peptide are associated with the development of polycystic ovary syndrome. J Clin Endocrinol Metab. 2014;99(12):E2539-48.

9. Zheng Y, Ley SH, Hu FB. Global aetiology and epidemiology of type 2 diabetes mellitus and its complications. Nat Rev Endocrinol. 2018;14(2):88-98.
10. Ahrén B. Use of DPP-4 inhibitors in type 2 diabetes: focus on sitagliptin. Diabetes Metab Syndr Obes. 2010;3:31-41.

11. Liu $\mathrm{Y}, \mathrm{Xu}$ F, Jiang P. Effect of sitagliptin on expression of skeletal muscle peroxisome proliferator-activated receptor $\gamma$ coactivator- $1 \alpha$ and irisin in a rat model of type 2 diabetes mellitus. J Int Med Res. 2020;48(5):300060519885569.

12. Classification I. Standards of medical care in diabetes-2014. Diabetes Care. 2014;37(Suppl 1):S14-80.

13. Katsuki A, Sumida Y, Gabazza EC, et al. Homeostasis model assessment is a reliable indicator of insulin resistance during follow-up of patients with type 2 diabetes. Diabetes Care. 2001;24(2):362-5.

14. Bermúdez V, Cano R, Cano $\mathrm{C}$, et al. Homeostasis model assessment (HOMA) as surrogate insulinization criteria in patients with type 2 diabetes. Am J Ther. 2008;15(4):409-16.

15. Xiang L, Xiang G, Yue L, Zhang J, Zhao L. Circulating irisin levels are positively associated with endothelium-dependent vasodilation in newly diagnosed type 2 diabetic patients without clinical angiopathy. Atherosclerosis. 2014;235(2):328-33.

16. Zhang $\mathrm{M}$, Chen $\mathrm{P}$, Chen $\mathrm{S}$, et al. The association of new inflammatory markers with type 2 diabetes mellitus and macrovascular complications: a preliminary study. Eur Rev Med Pharmacol Sci. 2014;18(11):1567-72.

17. Liu JJ, Wong MDS, Toy WC, et al. Lower circulating irisin is associated with type 2 diabetes mellitus. J Diabetes Complications. 2013;27(4):365-9.

18. Huerta-Delgado AS, Roffe-Vazquez DN, GonzalezGil AM, et al. Serum irisin levels, endothelial dysfunction, and inflammation in pediatric patients with type 2 diabetes mellitus and metabolic syndrome. J Diabetes Res. 2020;2020:1949415.

19. Sanchis-Gomar F, Perez-Quilis C. The p38-PGC$1 \alpha$-irisin-betatrophin axis: exploring new pathways in insulin resistance. Adipocyte. 2014;3(1):67-8.

20. Zhang Y, Li R, Meng Y, et al. Irisin stimulates browning of white adipocytes through mitogenactivated protein kinase p38 MAP kinase and ERK MAP kinase signaling. Diabetes. 2014;63(2):514-25.

21. Huh JY, Mougios V, Kabasakalis A, et al. Exerciseinduced irisin secretion is independent of age or fitness level and increased irisin may directly modulate muscle metabolism through AMPK activation. J Clin Endocrinol Metab. 2014;99(11): E2154-61. 
22. Scott R, Loeys T, Davies MJ, Engel SS. Efficacy and safety of sitagliptin when added to ongoing metformin therapy in patients with type 2 diabetes. Diabetes, Obes Metab. 2008;10(10):959-69.

23. Takihata M, Nakamura A, Tajima $K$, et al. Comparative study of sitagliptin with pioglitazone in Japanese type 2 diabetic patients: the COMPASS randomized controlled trial. Diabetes Obes Metab. 2013;15(5):455-62.

24. Shigematsu E, Yamakawa T, Kadonosono K, Terauchi Y. Effect of sitagliptin on lipid profile in patients with type 2 diabetes mellitus. J Clin Med Res. 2014;6(5):327-35.

25. Herman WH, Dungan KM, Wolffenbuttel BHR, et al. Racial and ethnic differences in mean plasma glucose, hemoglobin A 1c, and 1,5-anhydroglucitol in over 2000 patients with type 2 diabetes. J Clin Endocrinol Metab. 2009;94(5):1689-94.

26. Deacon CF. Physiology and pharmacology of DPP-4 in glucose homeostasis and the treatment of type 2 diabetes. Front Endocrinol (Lausanne). 2019;10:80.

27. Kulkarni RN, Brüning JC, Winnay JN, Postic C, Magnuson MA, Kahn CR. Tissue-specific knockout of the insulin receptor in pancreatic $\beta$ cells creates an insulin secretory defect similar to that in type 2 diabetes. Cell. 1999;96(3):329-39.

28. Shirakawa J, Amo K, Ohminami H, et al. Protective effects of dipeptidyl peptidase-4 (DPP-4) inhibitor against increased $\beta$ cell apoptosis induced by dietary sucrose and linoleic acid in mice with diabetes. J Biol Chem. 2011;286(29):25467-76.

29. Duan H, Ma B, Ma X, et al. Anti-diabetic activity of recombinant irisin in STZ-induced insulin-deficient diabetic mice. Int J Biol Macromol. 2016;84:457-63.

30. Lee HJ, Lee JO, Kim N, et al. Irisin, a novel myokine, regulates glucose uptake in skeletal muscle cells via AMPK. Mol Endocrinol. 2015;29(6):873-81.

31. de la Iglesia R, Lopez-Legarrea P, Crujeiras AB, et al. Plasma irisin depletion under energy restriction is associated with improvements in lipid profile in metabolic syndrome patients. Clin Endocrinol (Oxf). 2014;81(2):306-11.

32. Huh JY, Siopi A, Mougios V, Park KH, Mantzoros CS. Irisin in response to exercise in humans with and without metabolic syndrome. J Clin Endocrinol Metab. 2015;100(3):E453-7. 